\title{
Bioinformatics Study of Plasma Differentially Expressed Proteins in H-Type Hypertension with or without Acute Cerebral Infarction
}

\author{
Nanyao Chen', Min Guo ${ }^{2 *}$, Dan Yu ${ }^{3}$, Feng Zhou ${ }^{3}$ \\ ${ }^{1}$ The Fourth People's Hospital of Haikou, Haikou, China \\ ${ }^{2}$ Hainan General Hospital, Haikou, China \\ ${ }^{3}$ Central South University Xiangya School of Medical Affiliated Haikou Hospital, Haikou, China \\ Email: ^g2002m@163.com
}

How to cite this paper: Chen, N.Y., Guo, M., Yu, D. and Zhou, F. (2021) Bioinformatics Study of Plasma Differentially Expressed Proteins in H-Type Hypertension with or without Acute Cerebral Infarction. Journal of Behavioral and Brain Science, 11, 1-9.

https://doi.org/10.4236/jbbs.2021.111001

Received: November 9, 2020

Accepted: December 28, 2020

Published: December 31, 2020

Copyright $\odot 2021$ by author(s) and Scientific Research Publishing Inc. This work is licensed under the Creative Commons Attribution International License (CC BY 4.0).

http://creativecommons.org/licenses/by/4.0/

\begin{abstract}
Objective: To investigate the bioinformatics of differentially expressed proteins in plasma in patients with acute cerebral infarction associated with H-type hypertension. Methods: Gene chip public database (gene expression omnibus, GEO) GDS4521 chip data, in the chip in 30 cases of $H$ patients with acute cerebral infarction with high blood pressure and age, gender, matching the $20 \mathrm{H}$ hypertension patients as the research object, collects the mononuclear cells (PBMCs) is used to detect the gene chip, using the GO (gene Ontology, GO), protein function analysis tools such as KEGG, screening and analysis of enrichment of differentially expressed genes function and related signaling pathway. Results: 31 genes in PBMCs were significantly changed in h-type hypertension and H-type hypertension with acute cerebral infarction, 32 of which were increased and 2 of which were decreased. GO analysis showed that in terms of biological processes, the genes related to inflammatory response and neutrophil chemotaxis were the most. In terms of molecular function, chemokine activity-related genes are the most. KEGG signaling pathway analysis showed that the most differentially expressed genes were located in the TNF signaling pathway. Conclusion: H-type hypertension with acute cerebral infarction has a variety of functional proteins and signaling pathways changes, suggesting that inflammatory response in H-type hypertension with acute cerebral infarction recovery period may still play a role in the prognosis and reactivation of the disease.
\end{abstract}

\section{Keywords}

Acute Cerebral Infarction, Proteomics, ITRAQ Technology,

Immunohistochemical 


\section{Introduction}

Cerebrovascular disease has become the first cause of disability and death among urban and rural population in China, and its incidence tends to increase year by year. Cerebral infarction, also known as ischemic stroke, is mainly divided into hemorrhagic and ischemic types, which have obvious differences in pathogenesis, pathophysiological mechanism, clinical rehabilitation, prognosis and other aspects. Epidemiological studies have shown that there are 1.5 million to 2 million new cases of stroke every year in China, the annual incidence of stroke after age adjustment is (116/219)/100,000 population, and the annual mortality of stroke is $(56-142) / 100,000$ population [1]. In recent years, "omics" techniques for the analysis of various biomolecules have attracted extensive attention [2] [3] [4] [5] [6]. As a systematic collection of individuals of some species, omics, for example, the combination of all the proteins of the organism, namely proteomics, and the combination of all the metabolites of the organism, namely metabolomics, has become an important method of reducing the nature of phenomena [7]. At present, transcriptomics, proteomics and the extended metabolomics and polypeptide omics are studied more maturely. Proteomics is a holistic approach to the study of cells and tissues or the proteome of organisms. It was first proposed by Wilkins and Williams in 1994 [8]. Proteomics provides a good breakthrough point for the study of the occurrence and development of acute cerebral infarction. ITRAQ is a high-throughput, high-sensitivity protein quantitative technique, which is widely used in the medical field [9]. Compared with other previous proteomics technologies, it can effectively detect differentially expressed proteins between different groups, and reduce experimental errors, etc. [10]. Cerebrovascular disease is an important disease that leads to human disability. The pathological process involves different types of nerve cells, blood-brain barrier and blood circulation disorder, and the clinical phenotypes are diverse [11]. Proteomics is a large-scale study of protein expression levels, post-translational modifications, and protein-protein interactions [12]. It can dynamically, comprehensively and quantitatively investigate the changes of protein types and quantities in the process of disease development, and can complete the extremely complex research on protein function and cytokine changes. Aging significantly affects the vascular components of the neurovascular unit. Old age reduces the ability of endothelial cell regeneration and the number of endothelial progenitor cells, thus reducing the number of capillaries. With age, the glial cells' ability to repair the central nervous system is weakened. These structural and functional changes increase the likelihood of stroke and increase the degree of brain injury in the elderly after stroke. Hyperlipidemia, diabetes and hypertension increase the level of blood-brain barrier damage. Hyperlipidemia increases lipid peroxidation, thereby increasing protease activity, downregulating the expression of tightly connected related proteins, and increasing inflammation are all possible mechanisms that aggravate the damage of bloodbrain barrier [13]. Hypertension is closely related to tight junction function, 
blood-brain barrier and cerebral edema. Diabetes also damages the blood-brain barrier. In particular, the fluctuation of blood glucose concentration will change the transport direction of the blood-brain barrier, affect the stability of tight connections, and increase the oxidative stress response [14]. Hyperglycemia also aggravates inflammation and causes damage to neurovascular units. In addition, the components of neurovascular units, such as endothelial cells, pericytes and podocytes, are also affected by risk factors. This study using public microarray database GEO quantitative proteomic techniques [15] for differences in protein spectrum analysis, qualitative and quantitative results by the relevant differences in protein, and these differences in protein function, KEGG pathway analysis, further confirm the accuracy of protein spectrum experiment, from the view point of system biology height and histology to accurate validation and interpretation $\mathrm{H}$ hypertension with or without development of acute cerebral infarction, occur, and surgical treatment, the relationship between the functional recovery and prognosis judgement. To identify the two differential proteins at the same time to distinguish them from etiological targets. It will bring a broad prospect for the etiology, early warning, diagnosis and treatment, new methods and techniques for prognosis and the establishment of an assessment and analysis system for this disease. At the same time, the differential protein of emergency expression in the early stage of acute cerebral infarction was identified to distinguish from the etiological target. To promote the disciplinary development of this field through substantive interdisciplinary research.

\section{Materials and Methods}

\subsection{Object}

To set diagnostic criteria, refer to the appropriate guidelines or expert consensus: $\mathrm{H}$-type hypertension is diagnosed as hypertension with hyperhomocysteine. The diagnosis of acute cerebral infarction was based on CT or MRI images. Acute cerebral infarction associated with $\mathrm{H}$-type hypertension is acute cerebral infarction with $\mathrm{H}$-type hypertension. Healthy controls excluded the diagnosis of these diseases.

Criteria for inclusion: All participants are Han Chinese, native of Hainan province, and have lived here for a long time. $\mathrm{H}$-type hypertension-related acute cerebral infarction group: the inclusion time was within 24 hours after onset without thrombolytic therapy. H-type hypertension group: from the physical examination center, no history of other basic diseases, enrolled in the last 2 weeks only take antihypertensive drugs and/or B vitamins. (3) Healthy control group: from the physical examination center, no history of basic diseases, no history of taking medicine in the last 2 weeks. Exclusion criteria: age $<50$; Transient ischemic attack; Hemorrhagic stroke; Traumatic brain injury; Stroke caused by brain tumor; Lung, heart, liver and kidney diseases and their complications. The information of clinical patients and healthy physical examinators meeting the experimental conditions was collected. $5 \mathrm{ml}$ of whole venous blood 
was collected by EDTA anticoagulant tube, and the plasma and mononuclear cells were centrifuged and stored at $-80^{\circ} \mathrm{C}$, respectively. All the enrolled patients signed informed consent forms, and the research project was approved by the Ethics Committee of Human Research in Haikou No. 4 People's Hospital in 2018.

\subsection{The Selection of Chip Data and the Grouping of Research Objects}

GDS4521 chip data in Gene Expression Omnibus (GEO) was selected. Peripheral blood mononuclear cells (PBMCs) were collected from 30 patients with H-type hypertension and acute cerebral infarction, including 17 males and 13 females, with an average age of $(61.02 \pm 9.16)$ years. Twenty patients with $\mathrm{H}$-type hypertension were taken as the control group, including 11 males and 9 females with an average age of $(60.23 \pm 12.15)$ years old. Family history of stroke, severe anemia, and active anaphylaxis was excluded. There were no statistically significant differences in gender composition, age, combined with basic diseases such as hypercholesterolemia and diabetes, as well as lifestyle habits such as smoking and drinking.

\subsection{Methods}

GEO2R is a web-based interaction tool that analyzes two or more gene chip samples from the GEO database to obtain genes that are differentially expressed under certain experimental conditions. GO, KEGG, DAVID, G: Profiler and other online analysis tools are commonly used in bioinformatics analysis, which can carry out gene ontology GO enrichment analysis, KEGG signal pathway analysis, functional annotation and so on for a large number of genes. These tools were used for gene function annotation, enrichment analysis and signal pathway analysis of the screened differentially expressed genes. The String Database (String 10.0) is an online analysis tool used to predict functional correlations between proteins, using this tool to predict proteins interacting with differentially expressed genes found.

\section{The Results}

\subsection{Changes of PBMCs Protein Expression in the Two Groups}

GDS4521 chip data in GEO database were collected and analyzed by GEO2R to compare the gene expression of PBMCs in H-type hypertension patients with acute cerebral infarction and $\mathrm{H}$-type hypertension patients, so as to value means difference. A difference of more than two times was considered as the standard, and 53 differentially expressed genes were obtained. After the deletion of repeat and no geneID genes, 37 differentially expressed genes (PTGS2, CCNL1, SAMSN1, TMEM107, SNORD3D, VIM, JUN, CXCL8, G0S2, LINC00936, NAMPT, EGR1, OSM, BRE-AS1, SOCS3, FOSB, NR4A2, PPP1R15A, CD83, EREG, IL1B, ATF3, TNFAIP3) were finally screened out CXCL2, CCL3L3, DUSP2, RGS1, CXCL1, 
CCL20, CXCL3, PHLDA1, IER3, SOD2, TNF, EIF5A, HLA-DQA1, TNFRSF17) were up-regulated in 34 patients with Type $\mathrm{H}$ hypertension and acute cerebral infarction, and down-regulated in 3 types (EIF5A, HLA-DQA1, TNFRSF17) (Figure 1).

\subsection{Functional Enrichment and Signal Pathway Analysis of Differentially Expressed Genes}

GO analysis of the screened differentially expressed genes showed that, in terms of biological processes, the differentially expressed genes were divided into 51 categories, including 9 categories containing more than 5 genes, mainly focusing on inflammatory response and immune response, chemotaxis, cell proliferation and apoptosis, etc. In terms of cell composition, the differentially expressed genes were mainly distributed in cytoplasm and extracellular space. In terms of molecular function, differentially expressed genes can be divided into 6 categories, mainly focusing on chemokine activity and receptor binding, transcription factor activity, and cytokine activity, with chemokine activity-related genes being the most. In this study, GO functional analysis showed that most of the functions were expressed by multiple gene variants, which indirectly confirmed that $\mathrm{H}$-type hypertension with acute cerebral infarction is a complex disease caused by the action of multiple genes. KEGG signaling pathway analysis showed that the differentially expressed genes were mainly distributed in tumor necrosis factor

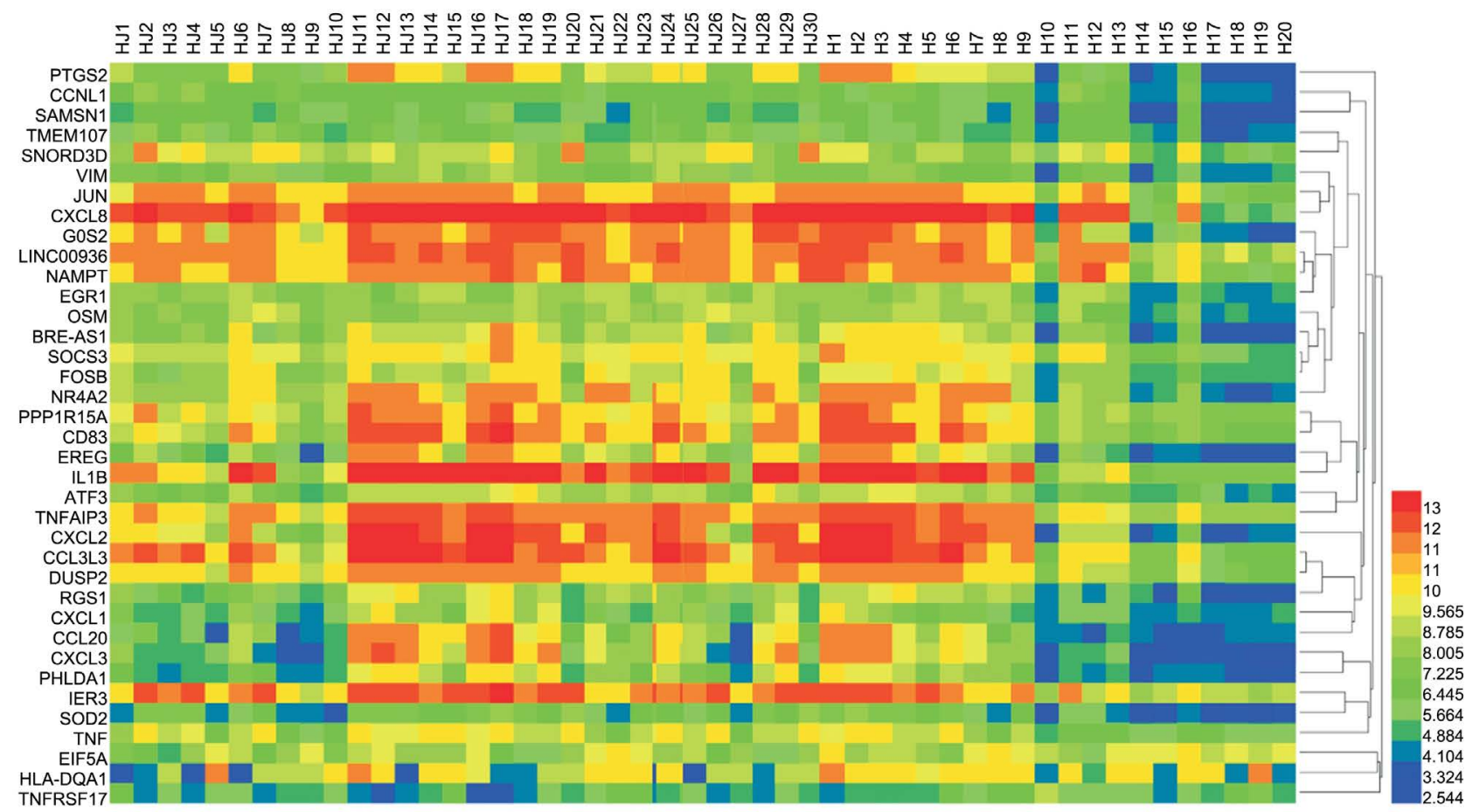

Figure 1. Heat map of differentially expressed PBMCs between HJ patients and control group. Note: HJ: Type H hypertension with acute cerebral infarction, PBMCs: peripheral blood mononuclear cells; The differential gene expression was represented from high to low in red to blue, the left side of the heat map was the gene name, the top was the sample number (HJ was the group of $\mathrm{H}$-type hypertension with acute cerebral infarction, $\mathrm{H}$ was the control group), and the tree structure on the right side represented the similarity clustering relationship between the samples. 
(TNF- $\alpha$ ), nuclear transcription factor KB (NF-KB), chemokines, Toll-likereceptor (Toll-Likereceptor), NOD receptor and other pathways, among which the most related genes were in TNF- $\alpha$ signaling pathway. The selected differential protein ids were converted into Uniprot IDS, and then the six differential protein ids were input into the omes-Bean online analysis platform to obtain the corresponding bioinformation analysis results. It showed that 175 biological processes were involved, among which 96 were of significant significance. Seventeen cell components were involved, 6 of which were significant. There were 36 molecular functions involved, 20 of which were significant. Four differentially expressed protein KEGG pathways were involved, including 1 complement pathway and 1 coagulation cascade pathway closely related to cerebral ischemia. Single-celled organisms for cell processes, to the process, biological control, metabolic process, to stimulate the reaction process, the process of multicellular organisms, positioning process, cell segmentation groups or biosynthesis, signal process, development process, the process of the immune system, mobile process, biological process, biological adhesive process, growth, proliferation, cell destruction process of combination, catalytic activity, enzyme activities, regulating the activity of the molecular structure, turn the activity, transduction activity, receptor activity, antioxidant activity, protein binding activity of transcription factors, electronic carrier activity, channel regulation activity, nucleic acids in combination with the activity of transcription factors, Chemical inducer activity, translational regulatory activity, cells, organelles, plasma membrane, intercellular regions, macromolecular complexes, membrane proteins, extracellular matrix, cellular adhesion, synapses, nuclei, as shown in Figure 2.

\subsection{Interaction Analysis of Differentially Expressed Genes}

Except for the 9 proteins with interaction predicted, the other differential proteins mainly formed two networks, with TNF and JUN genes as the center, respectively. Discuss Protein is an important marker of pathophysiological changes of diseases [16]. However, studies on human acute cerebral infarction are limited by sample sources, difficulty in obtaining brain tissue and cerebrospinal fluid samples from patients with acute cerebral infarction, and the impossibility of approving corresponding clinical trials by ethics committees. After

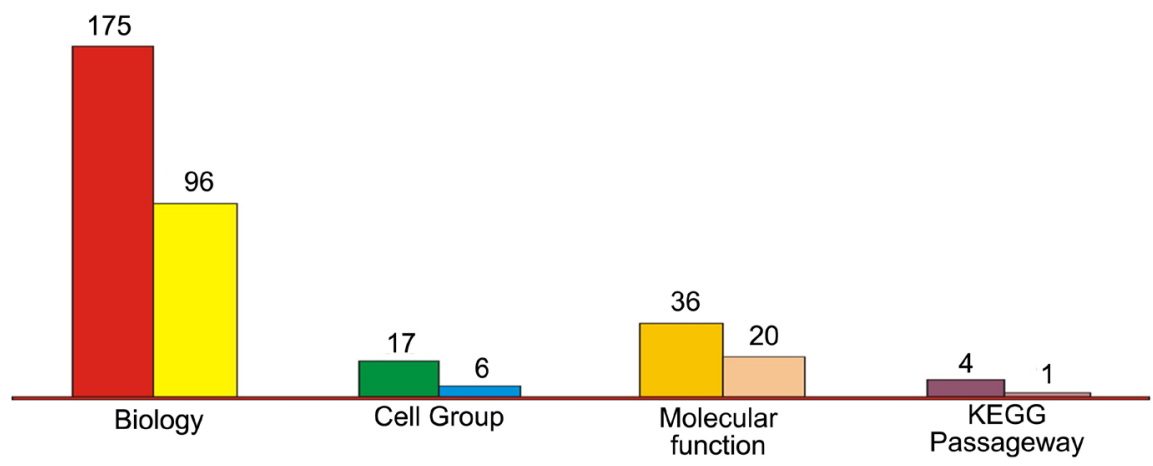

Figure 2. Differential protein point mass spectrometry. 
acute cerebral infarction, the blood-brain barrier is destroyed, and the specific proteins closely related to acute cerebral infarction are produced in the brain and may enter the circulatory system. Plasma proteome reflects the pathophysiological state before and after the change of disease, which is an important window to understand the molecular changes before and after acute cerebral infarction. Proteomics is the comprehensive and holistic study of the biological functions of proteins, the complete and quantitative proteome mapping of a disease state, including the localization of proteins in cells, the reconstruction of their network structures and complexes, and the depiction of signaling pathways and modifications of translated proteins. With the development of proteomics research techniques, we can study different samples of various disease states to obtain global and comprehensive differentially expressed proteins [17]. In particular, the iTRAQ technique has high sensitivity to allow mass spectrometers to analyze multiple groups of samples at once and to identify low abundance proteins. Some proteomic studies on acute cerebral infarction have deepened our understanding of the pathophysiological changes of cerebral infarction. Through quantitative analysis of a large number of circulating metabolites by proteomics method, metabolic changes can be identified in advance before the occurrence of acute cerebral infarction, so as to understand the pathophysiological mechanism of the disease as a whole. Through the reanalysis of PBMCs biochip in patients with acute cerebral infarction, the 10 differentially expressed proteins with or without acute cerebral infarction are CCL20, CXCL1, PTGS2, TNFAIP3, TNF, CCL3L3, CXCL2, CXCL3, IL1B and CXCL8, and 10 differentially expressed proteins with or without acute cerebral infarction are closely related to immune response, namely RGS1, CCL20, HLA-DQA1, CXCL1, TNF, OSM, CXCL2, CXCL3, IL1B and CXCL8. Although there have been many studies on the role of some of these proteins in the early pathological changes of acute cerebral infarction, such as CXCL1, PTGS2 and TNF, the exact role of these proteins in the recovery stage remains unclear. At the same time, the role of some differential proteins in acute cerebral infarction is rarely studied or not yet involved, such as CCL20, TNFAIP3, CCL3L3, CXCL3, RGS1, and HLA-DQA1. The results of the interaction network of differentially expressed genes also found that the above proteins were closely related to each other, suggesting that these proteins and their related signaling pathways may become new targets in the pathogenesis and treatment of acute cerebral infarction, and may also become markers to predict the recurrence of acute cerebral infarction, which is worth further research and verification. Several significantly different proteins, complement and coagulation cascade pathways are the key processes for the occurrence of cerebral ischemia injury. Starting from this pathway may help to elucidate the mechanism of the occurrence and development of cerebral infarction, and may also reveal the key targets of drug therapy. In addition, the downstream pathway of this pathway may also affect the key points of cerebral infarction. Of course, cerebral infarction is a complex pathological process, and there must be other proteins and regulatory mechanisms involved, which still need further in-depth study. Its 
exact clinical significance needs further study.

\section{Conclusion}

To sum up, through bioinformatics method, this study further analyzes the $\mathrm{H}$ hypertension patients with acute cerebral infarction recovery period with PBMCs gene chip, screen out several differentially expressed genes, and find some possible acute cerebral infarction restore stability research new target molecules and signaling pathways, for resuming the stabilization treatment of acute cerebral infarction and recurrence prediction provides a new way of thinking.

\section{Foundation Project}

Supported by Hainan Natural Science Foundation (Project No. 818MS161).

\section{Conflicts of Interest}

The authors declare no conflicts of interest regarding the publication of this paper.

\section{References}

[1] Writing Group of Secondary Prevention Guidelines for Ischemic Stroke, Cerebrovascular Disease Group, Neurology Branch of Chinese Medical Association (2010) Chinese Guidelines for Secondary Prevention of Ischemic Stroke and Transient Ischemic Attack 2010. Chinese Journal of Neurology, 43, 154-160.

[2] Prentice, R.L., Paczesny, S., Aragaki, A., et al. (2010) Novel Proteins Associated with Risk for Coronary Heart Disease or Stroke among Postmenopausal Women Identified by In-Depth Plasma Proteome Profiling. Genome Medicine, 2, 48. https://doi.org/10.1186/gm169

[3] Huang, Z., Lin, L., Gao, Y., et al. (2011) Bladder Cancer Determination via Two Urinary Metabolites: A Biomarker Pattern Approach. Molecular \& Cellular Proteomics, 10, M111.007922. https://doi.org/10.1074/mcp.M111.007922

[4] Sung, J.H., Shah, F.A., Gim, S.A., et al. (2016) Identification of Proteins in Hyperglycemia and Stroke Animal Models. Journal of Surgical Research, 200, 365-373. https://doi.org/10.1016/j.jss.2015.07.020

[5] Otero-Ortega, L., Gutiérrez-Fernández, M., Ramos-Cejudo, J., et al. (2015) White Matter Injury Restoration after Stem Cell Administration in Subcortical Ischemic Stroke. Stem Cell Research \& Therapy, 6, 121. https://doi.org/10.1186/s13287-015-0111-4

[6] Lopez, M.F., Krastins, B., Sarracino, D.A., et al. (2015) Proteomic Signatures of Serum Albumin-Bound Proteins from Stroke Patients with and without Endovascular Closure of PFO Are Significantly Different and Suggest a Novel Mechanism for Cholesterol Efflux. Clinical Proteomics, 12, 2. https://doi.org/10.1186/1559-0275-12-2

[7] Lindsey, M.L., Mayr, M., Gomes, A.V., et al. (2015) Transformative Impact of Proteomics on Cardiovascular Health and Disease: A Scientific Statement from the American Heart Association. Circulation, 132, 852-872. https://doi.org/10.1161/CIR.0000000000000226

[8] Wasinger, V.C., Cordwell, S.J., Cerpa-Poljak, A., et al. (1995) Progress with 
Gene-Product Mapping of the Mollicutes: Mycoplasma genitalium. Electrophoresis, 16, 1090-1094. https://doi.org/10.1002/elps.11501601185

[9] Siegel, R.L., Miller, K.D. and Jemal, A. (2016) Cancer Statistics, 2016. CA: A Cancer Journal for Clinicians, 66, 7-30. https://doi.org/10.3322/caac.21332

[10] Chan, B.A. and Coward, J.I. (2013) Chemotherapy Advances in Small-Cell Lung Cancer. Journal of Thoracic Disease, 5, S565.

[11] Silver, M.H., Foidart, J.M. and Pratt, R.M. (1981) Distribution of Fibronectin and Collagen during Mouse Limb and Palate Development. Differentiation, 18, 141-149. https://doi.org/10.1111/j.1432-0436.1981.tb01115.x

[12] Jielile, J. (2008) Biomechanics during Achilles Tendon Healing. Journal of Clinical Rehabilitative Tissue Engineering Research, 12, 8352-8357.

[13] Cai, L., Zhang, L., Liu, A., et al. (2012) Prevalence, Awareness, Treatment, and Control of Dyslipidemia among Adults in Beijing, China. Journal of Atherosclerosis and Thrombosis, 19, 159-168. https://doi.org/10.5551/jat.10116

[14] Kiran, M. and Nagarajaram, H.A. (2013) Global versus Local Hubs in Human Protein-Protein Interaction Network. Journal of Proteome Research, 12, 5436-5446. https://doi.org/10.1021/pr4002788

[15] Edgar, R., Domrachev, M. and Lash, A.E. (2002) Gene Expression Omnibus: NCBI Gene Expression and Hybridization Array Data Repository. Nucleic Acids Research, 30, 207-210. https://doi.org/10.1093/nar/30.1.207

[16] Crinò, L., Weder, W., van Meerbeeck, J., et al. (2010) Early Stage and Locally Advanced (Non-Metastatic) Non-Small-Cell Lung Cancer: ESMO Clinical Practice Guidelines for Diagnosis, Treatment and Follow-Up. Annals of Oncology, 21, v103. https://doi.org/10.1093/annonc/mdq207

[17] Barrett, T., Wilhite, S.E., Ledoux, P., et al. (2013) NCBI GEO: Archive for Functional Genomics Data Sets-Update. Nucleic Acids Research, 41, D991-D995. https://doi.org/10.1093/nar/gks1193 